\title{
Optimización del proceso de obtención de un adsorbente natural a partir de corteza de pino
}

\author{
Marlon Manuel Cuetocue-Petins ${ }^{1 *}$; Rodrigo Andrés Sarria-Villa ${ }^{1}$; José Antonio Gallo-Corredor ${ }^{1}$ \\ Ricardo Benítez-Benitez ${ }^{2}$ \\ ${ }^{1}$ Grupo de Investigación en Química Analítica Ambiental (GIQA); ${ }^{2}$ Química de Productos Naturales (QPN), \\ Departamento de Química. Universidad del Cauca, carrera 3 n. ${ }^{\circ} 3 \mathrm{~N}-100$, Popayán. Colombia. \\ *petins@unicauca.edu.co
}

\section{Resumen}

Se han extraído los taninos presentes en la corteza de Pinus patula para desarrollar un adsorbente mediante una reacción de reticulación, que se da entre los grupos fenólicos debido al tratamiento con ácido sulfúrico, que es ampliamente utilizado como agente reticulante de grupos polifenólicos presentes en los taninos. En el presente estudio, el diseño de Box-Behnken se usó para maximizar el rendimiento de la reacción de reticulación y, por lo tanto, obtener las mejores propiedades mecánicas del adsorbente preparado. La variable de respuesta estudiada fue el rendimiento porcentual de la reacción, y las variables independientes evaluadas en el diseño Box-Behnken incluyeron, relación Sólido/Líquido (gramos de tanino/mL de $\left.\mathrm{H}_{2} \mathrm{SO}_{4}\right)\left(0,2-0,5 \mathrm{~g} \cdot \mathrm{mL}^{-1}\right)$, temperatura $\left(75-95^{\circ} \mathrm{C}\right)$ y tiempo $(8-12 \mathrm{~h})$. El modelo estadístico permitió estimar un rendimiento porcentual óptimo del $73,81 \%$, que se obtiene con una relación S/L de 0,499 $\mathrm{gmL}^{-1}$, temperatura de $95{ }^{\circ} \mathrm{C}$ y tiempo de reacción de 10,45 h. La modificación de los grupos funcionales superficiales de los taninos fue establecida por medio de los espectros IR. El adsorbente presentó un área superficial BET de $4,17 \mathrm{~m}^{2} \mathrm{~g}^{-1}$.

Palabras clave: Taninos; Reticulación; Adsorbente. 


\title{
Optimization of the process of obtaining a natural pine bark adsorbent
}

\begin{abstract}
The tannins present in the bark of Pinus patula have been extracted to develop an adsorbent through a crosslinking reaction, which occurs between the phenolic groups due to treatment with sulfuric acid, which is used as a crosslinking agent for polyphenolic groups present in the tannins. In the present study, the Box-Behnken design is used to maximize the performance of the crosslinking reaction and, therefore, to obtain the best mechanical properties of the prepared adsorbent. The response variable studied was the percentage yield of the reaction, and the independent variables evaluated in the Box-Behnken design included, Solid/Liquid ratio (grams of tannin/mL of $\left.\mathrm{H}_{2} \mathrm{SO}_{4}\right)\left(0.2-0.5 \mathrm{gmL}^{-1}\right)$, temperature $\left(75-95^{\circ} \mathrm{C}\right.$ ) and time (8-12 h). The verified statistical model estimates an optimal percentage yield of $73.81 \%$, it is obtained with an $\mathrm{S} / \mathrm{L}$ ratio of $0.499 \mathrm{gmL}^{-1}$, temperature of $95{ }^{\circ} \mathrm{C}$ and reaction time of $10.45 \mathrm{~h}$. The modification of the surface functional groups of the tannins was established by means of the IR spectra. The adsorbent had a BET surface area of $4.17 \mathrm{~m}^{2} \mathrm{~g}^{-1}$.
\end{abstract}

Keywords: Tannins; Crosslinking; Adsorbent.

\section{Otimização do processo de obtenção de um adsorvente natural de casca de pinheiro}

\begin{abstract}
Resumo
Os taninos presentes na casca de Pinus patula foram extraídos para desenvolver um adsorvente através de uma reação de reticulação, que ocorre entre os grupos fenólicos devido ao tratamento com ácido sulfúrico, usado como agente de reticulação para grupos polifenólicos presentes nos taninos. No presente estudo, o projeto Box-Behnken é utilizado para maximizar o desempenho da reação de reticulação e, portanto, para obter as melhores propriedades mecânicas do adsorvente preparado. A variável resposta estudada foi a porcentagem de rendimento da reação e as variáveis independentes avaliadas no projeto Box-Behnken incluíram a relação Sólido/Líquido (gramas de tanino/mL de $\left.\mathrm{H}_{2} \mathrm{SO}_{4}\right)\left(0,2-0,5 \mathrm{gmL}^{-1}\right)$, temperatura $\left(75-95^{\circ} \mathrm{C}\right)$ e tempo $(8-12 \mathrm{~h})$. O modelo estatístico verificado estima um rendimento percentual ideal de $73,81 \%$, é obtido com uma relação $\mathrm{S} / \mathrm{L}$ de $0,491 \mathrm{gmL}^{-1}$, temperatura de $95^{\circ} \mathrm{C}$ e tempo de reação de 10,45h. A modificação dos grupos funcionais da superfície dos taninos foi estabelecida por meio dos espectros de IR. O adsorvente tinha uma área de superfície BET de 4,17 $\mathrm{m}^{2} \mathrm{~g}^{-1}$.
\end{abstract}

Palavras-chave: Taninos; Reticulação; Adsorvente. 


\section{Introducción}

Los recursos naturales han constituido siempre las principales fuentes para el desarrollo de la economía de un país [1]. La biomasa forestal que se origina en el proceso de aserrado de la madera (fundamentalmente aserrín y corteza), constituye un material lignocelulósico que por su naturaleza química puede compararse con la del bagazo de caña y otros derivados de ésta. En los últimos años, se ha logrado un importante crecimiento y desarrollo en la tecnología, que ha traído como consecuencia una explotación cada vez más acelerada de estos recursos. Entre los recursos naturales se encuentran los residuos forestales, dentro de estos materiales se encuentran el aserrín y la corteza que resultan desechos de la industria de la elaboración primaria de la madera, constituidos por restos de aserríos, de ebanisterías y de plantaciones. El aserrín y la corteza son unos de los principales residuos forestales y su acumulación puede tener efectos ambientales negativos [1]. Que al descomponerse los mismos, se devuelva a la atmósfera el dióxido de carbono contenido en la materia orgánica, por la incidencia del sol y las altas temperaturas se provoca en los acumulados de aserrín una pirólisis a baja temperatura, producto de lo cual se emiten gases contaminantes al medio, estos residuos constituyen un medio ideal para la generación de plagas y enfermedades. Por lo tanto, transformar estos desechos en productos de uso social se traduce también como aplicación de tecnología para el saneamiento ambiental [2].

Las plantaciones forestales comerciales en Colombia, registradas ante el ICA a octubre de 2016 cubrían 310138 ha, concentradas principalmente en los departamentos de Antioquia, Meta, Vichada, Valle del Cauca y Córdoba. Las principales especies plantadas son de los géneros Pinus, Eucalyptus, Acacia y la teca (Tectona grandis) [3]. Según los registros de plantaciones comerciales, alrededor del $73 \%$ del área plantada presenta especies introducidas como pinos, el Pinus patula es la especie que mayor área de plantación presenta con 53 197,64 ha [4].

Los taninos son compuestos fenólicos poliméricos, producidos en las plantas como metabolitos y que tienen la capacidad de formar complejos con proteínas, polisacáridos, ácidos nucleicos, esteroides y saponinas. Los taninos son bien conocidos por ser polímeros naturales de bajo costo y omnipresentes, que se pueden extraer fácilmente de las plantas. Debido a su alto contenido de múltiples hidroxilos fenólicos adyacentes, los taninos exhiben una gran afinidad hacia muchos iones metálicos. Antes de su utilización, sin embargo, los taninos deben estar inmovilizados o reticulados debido a su solubilidad en agua. Algunas matrices insolubles en agua se han utilizado para la inmovilización de taninos como la celulosa y la agarosa. Sin embargo, los taninos se filtran fácilmente durante el proceso de adsorción debido a la asociación débil entre los taninos y las matrices [5]. Los taninos son excelentes candidatos para producir biosorbentes. Estos biopolímeros naturales ubicuos y económicos son de fácil extracción y conversión en insolubles (geles y espumas de tanino) o matrices inmovilizadas. Los adsorbentes a base de taninos (TBA) tienen una afinidad natural para absorber metales pesados, colorantes, tensioactivos y compuestos farmacéuticos de aguas contaminadas, y para acumular metales selectivos preciosos y críticos de las corrientes acuosas [6].

Recientemente ha habido un interés creciente en la biosorción, que es una propiedad de ciertos tipos de materiales de biomasa para unir y concentrar iones metálicos de soluciones acuosas [7]. En los mecanismos de la bioadsorción el proceso de acción es bastante complejo y básicamente difícil de ser entendido. Sin embargo, puede ser uno o una combinación de varios procesos que incluyen complejación, intercambio iónico, interacción electrostática, coordinación, quelación, adsorción y microprecipitación. En general, los iones metálicos se unen con los bioadsorbentes a través de ciertos grupos funcionales activos de ligandos tales como grupos carbonilo, carboxilo, fenólico o amino [8].

Es muy necesario entender cómo se une el ion metálico a la biomasa. Esto se logrará identificando los grupos funcionales activos que pueden unirse con iones metálicos. El carácter higroscópico y afinidad por el agua característica de los materiales biosorbentes permite que el agua penetre en la parte no cristalina de la celulosa, hemicelulosa y lignina. Por consiguiente, en un proceso de adsorción, las soluciones pueden interactuar con diferentes constituyentes de la pared celular. En la mayoría de los casos se producen fuertes enlaces químicos a través de reacciones selectivas, irreversibles y endotérmicas [8].

Estos materiales de biomasa son relativamente baratos y están disponibles en grandes cantidades en todo el mundo. El tanino condensado es bien 
conocido por ser un polímero natural económico, omnipresente y presenta muchos grupos hidroxilo. El tanino tiene altas afinidades por los metales, sin embargo, cuando se usa tanino como adsorbente, debe inmovilizarse porque las moléculas de tanino son solubles en agua [7]. Existen diferentes estrategias para modificar los taninos y así inmovilizarlos, entre ellas, una reacción gradual en presencia de un catalizador ácido o base obtenido mediante reacción con formaldehído da como resultado la insolubilización de taninos [9].

Se ha informado que el tanino insolubilizado tiene la capacidad de retener varios metales. Hay antecedentes que indican que el grupo ortodihidroxilo es el involucrado en la complejación metálica. La naturaleza aniónica de los grupos fenólicos, debido a su facilidad de desprotonación y estabilización por resonancia, también permite el establecimiento de interacciones electrostáticas entre los adsorbatos catiónicos y la superficie adsorbente negativa [10]. Puede acomplejar metales como el uranio, el plomo, y metales preciosos [7]. Se espera que el absorbente a base de tanino sea útil como adsorbente para la recuperación de metales preciosos.

Como el tanino de la corteza de pino es parcialmente soluble en agua, sus aplicaciones como adsorbente son limitadas. Por lo tanto, para superar este problema y mejorar las características de adsorción, se debe transformar en una matriz insoluble en agua, ya sea por reticulación o por inmovilización [11]. Debido a que dicha inmovilización es costosa y complicada, el enfoque más práctico es la reticulación. Se ha informado que el ácido sulfúrico concentrado es uno de los agentes de reticulación más efectivos para los residuos de biomasa [11]. La reticulación de materiales de biomasa con ácido sulfúrico concentrado bajo la influencia del calor da lugar a una reacción de condensación entre los grupos funcionales superficiales, en esta reacción están involucrados los grupos superficiales hidroxilo polifenoles [11]. Bajo condiciones ácidas fuertes, puede ocurrir hidrólisis o auto condensación. La degradación en medios ácidos conduce a la formación de catequinas y antocianinas. Un segundo tipo de reacción es la condensación después de la hidrólisis del heterociclo. Los iones de $p$-hidroxibencilcarbonio formados se condensan en los sitios nucleofílicos de otra unidad de tanino [12].
El Pinus patula presenta una madera blanda con aplicaciones en la industria de Colombia, en la cual se producen importantes cantidades de residuos forestales [13]. No hay datos específicos reportados sobre la producción actual de Pinus patula en la industria maderera [4]. En estudios de campo se estima que el proceso de obtención de madera genera $65 \%$ de residuos, estos residuos son principalmente cortezas de pino sin usos comerciales en Colombia [14]. A medio y largo plazo puede convertirse en un problema ambiental, además, hay una gran oportunidad económica para el aprovechamiento de estos residuos. Por lo tanto, los residuos como la corteza de pino pueden transformarse en diferentes productos de interés como biocombustibles, productos químicos y la gran tendencia en la extracción de sus componentes para ser empleados como adsorbentes [4].

En particular, en Colombia la corteza de Pinus Patula es un residuo forestal, abundante y económico, que con tratamientos adecuados puede utilizarse como alternativa para elaboración de adsorbentes convencionales. Por consiguiente, en esta investigación, se realizó la extracción de los taninos de la corteza de Pinus patula y su modificación por medio del proceso de reticulación para la obtención del gel y proyectar su potencial uso como adsorbente.

\section{Metodología}

\section{Obtención y adecuación de la corteza de Pinus patula}

Se utilizó entre 10 y $15 \mathrm{~kg}$ de corteza de Pinus patula adquiridos de la pinera de la empresa Smurfit Cartón de Colombia, ubicada en el municipio de Sotará, Cauca. La corteza fue sometida a un proceso de limpieza manual donde se retiraron diferentes residuos como polvo, ramas y hojas presentes en el material vegetal. Una vez limpia la corteza, se procedió a secarla a temperatura ambiente durante 20 días aproximadamente.

Reducción y clasificación por tamaños. Para obtener un tamaño de partícula apropiado para la extracción de los taninos, se usó un molino eléctrico de granos, hasta obtener partículas finas de la biomasa. La respectiva clasificación se llevó acabo en un tamiz ASTME-11-87 de malla № 60 y un tamaño de partícula de $250 \mu \mathrm{m}$. 
Caracterización fisicoquímica de la corteza de Pinus patula

Una vez acondicionado el tamaño de las partículas de la corteza se procedió a la respectiva caracterización, que consistió en la determinación de la humedad de acuerdo con la norma UNEEN 14774-3 (2010) y cenizas, de acuerdo con la norma UNE-EN 14775 (2010).

Humedad. El método gravimétrico es ampliamente utilizado para determinar el contenido de humedad y reconocido como un método primario. Se añadió $1 \mathrm{~g}$ de partículas pequeñas de la fracción retenida en el tamiz, la muestra se llevó a la estufa regulada a $103^{\circ} \mathrm{C}$ entre 8 y 10 horas, pasado este tiempo se colocó en un desecador hasta que se enfrió y luego se pesó. Este procedimiento se repitió hasta obtener peso constante [15].

Cenizas. La determinación del contenido de cenizas (\%p) se llevó a cabo por método gravimétrico, que consiste en llevar un gramo de corteza a un crisol y posteriormente a una mufla a $525^{\circ} \mathrm{C}$ durante un periodo de 4 horas [15].

\section{Extracción de los Taninos de la corteza de Pinus patula}

Para la extracción de los taninos de la corteza, se tomaron entre 50 y $150 \mathrm{~g}$ de corteza de pino previamente tamizada, y se adicionó entre 300 y $600 \mathrm{~mL}$ de agua desionizada y 3-7 g de $\mathrm{NaOH}$, la mezcla se agitó magnéticamente por $1 \mathrm{~h}$ a $90^{\circ} \mathrm{C}$. Posteriormente se filtró y la fracción líquida se secó en el horno entre 50 y $90{ }^{\circ} \mathrm{C}$ [15]. El porcentaje de extracción se determinó por la diferencia entre la cantidad de corteza utilizada y la cantidad de taninos obtenidos, una vez secos los taninos se maceraron en un mortero de porcelana para reducir el tamaño de partícula, posteriormente se tamizaron en un tamiz ASTME-11-87. № 60 con tamaño de partícula de $250 \mu \mathrm{m}$ [15].

\section{Diseño experimental para la reticulación de los} taninos extraídos de la corteza de Pinus patula Se determinaron los efectos de las condiciones de operación para llevar a cabo el proceso de reticulación de los taninos de corteza de pino mediante un diseño factorial completo $2^{3}$ y la posterior optimización para establecer las mejores condiciones experimentales a través de un diseño de superficie Box-Behnken. La elaboración y análisis de los diseños experimentales fueron realizados con el programa Statgraphics Centurión
XV.II. para diseñar los experimentos de la reacción de reticulación de los taninos y optimizar las condiciones operacionales para obtener el máximo rendimiento porcentual. Para establecer las variables dependientes e independientes de la reacción de reticulación de los taninos se tiene en cuenta los estudios realizados por Gurung et al. [5] empleando el árbol de Caqui.

Para establecer las condiciones óptimas de la síntesis del adsorbente, se llevó a cabo el diseño experimental de superficie de respuesta BoxBehnken, con tres factores y dos niveles (bajo y alto): relación $S / L\left(1 / 2 \mathrm{y} 1 / 5 \mathrm{gmL}^{-1}\right)$, temperatura $\left(75^{\circ} \mathrm{C}\right.$ y $\left.95^{\circ} \mathrm{C}\right)$ y tiempo ( $8 \mathrm{~h}$ y $12 \mathrm{~h}$ ) y como variable de respuesta el rendimiento de la reticulación de los taninos extraídos de la corteza de Pinus patula, establecido por la diferencia de masa antes y después de la reticulación de los taninos.

\section{Preparación del adsorbente a partir de los Taninos}

Para la reacción de reticulación de los taninos, se mezcló el extracto de taninos con ácido sulfúrico concentrado en un balón de fondo redondo de tres bocas de $250 \mathrm{~mL}$ y se agitó entre 8 y 12 horas a una temperatura entre 75 y $95{ }^{\circ} \mathrm{C}$, se utilizan las condiciones experimentales en los niveles establecidos en el diseño de Box-Behnken, con el propósito de optimizar el rendimiento porcentual de la reacción de reticulación para obtener geles de tanino. El producto negro tratado con ácido se neutralizó con solución de bicarbonato sódico y se filtró. La torta del filtro se lavó con agua desionizada y se secó en el horno a $70^{\circ} \mathrm{C}$. Finalmente, la masa seca se trituró para obtener polvo de partículas uniformes y se tamizó por un tamiz ASTME-11-87. $N^{\circ} 140$.

\section{Caracterización del adsorbente preparado}

Análisis instrumental. Los espectros fueron tomados en un espectrofotómetro marca Thermo ${ }^{\circledR R}$-NICOLET iS10 con pastilla de $\mathrm{KBr}$. Los espectros IR fueron analizados tanto para los taninos y los taninos después de la reticulación. El análisis BET para determinar el área superficial del adsorbente fue realizado por la Universidad del Valle (Colombia) en un equipo Nova 1000e, Quantachrome Instruments.

\section{Resultados y discusión}

Se determinaron características fisicoquímicas de la corteza de Pinus patula utilizada para la 
extracción de los taninos, que fueron utilizados para elaborar el adsorbente. Se determinó el contenido porcentual de humedad y cenizas de la corteza de Pinus patula. Los resultados experimentales obtenidos para el contenido porcentual de humedad y cenizas muestra valores de $26,27 \% \pm 0,62$ y $1,77 \% \pm 0,12$, respectivamente, los cuales se ajustan a los valores reportados en la literatura, debido a que el rango del porcentaje de humedad en la biomasa puede variar de $25-75 \%$, y de cenizas $1-2 \%$ [1].

Para la extracción de los taninos se empleó $\mathrm{NaOH}$ y 50-100 g de corteza de Pinus patula previamente reducida a un tamaño de partícula menor a 250 $\mu \mathrm{m}$. Los porcentajes de extracción alcanzaron un $47,32 \pm 0,85 \%$, valores muy similares a estudios realizados por Saravia Molina et al. [16].

\section{Optimización de las condiciones operacionales para la reticulación de los taninos}

En la tabla 1, se muestran los resultados obtenidos del análisis de varianza (ANOVA), realizado con ayuda del software Statgraphics Centurión XVI. II. El análisis de varianza se llevó a cabo para determinar la significancia estadística de cada efecto comparando su cuadrado medio contra un estimado del error experimental, de esta manera se obtuvieron los valores de los factores significativos para el rendimiento del proceso de reticulación de los taninos de corteza de Pinus patula. Para la optimización de las condiciones operacionales para la reticulación y establecer la significancia estadística de los efectos se utilizó un nivel confianza del 95\% (error máximo permisible $5 \%$ ), por lo tanto, se aceptaron como significativos aquellos efectos o parámetros que tengan un error (valor $\mathrm{P}$ ), menor que 0,05 .

Para analizar simultáneamente el efecto de los factores seleccionados, se plantearon las siguientes hipótesis que ayudaran a establecer las condiciones óptimas para la reticulación. Se plantearon dos hipótesis para cada uno de los efectos principales A (Relación S/L), B (Temperatura) y C (Tiempo).

- $\mathrm{H}_{0}$ : no hay un efecto debido al factor relación $S / L(A)$ en el porcentaje de reticulación de los taninos.

$\mathrm{H}_{1}$ : al menos hay un efecto debido al factor $\mathrm{A}$ en el porcentaje de reticulación de los taninos.

De igual forma se plantearon dos hipótesis para la interacción de los factores, la interacción $A B, A C$ y BC.

- $\mathrm{H}_{0}$ : no hay efecto de la interacción entre los factores $A$ y $B$ en el porcentaje de reticulación de los taninos.

$\mathrm{H}_{1}$ : hay efecto de la interacción entre los factores $\mathrm{A}$ y $\mathrm{B}$ en el porcentaje de reticulación de los taninos.

Así mismo se analizan las diferentes interacciones.

Una vez obtenidos los porcentajes de rendimiento de la reticulación de los taninos, ejecutando los experimentos estipulados mediante el diseño experimental de superficie de respuesta BoxBehnken, con tres factores y dos niveles (bajo y alto), se realizó el análisis de varianza ANOVA mostrado en la tabla 1.

Tabla 1. Análisis de varianza para el rendimiento.

\begin{tabular}{cccccc}
\hline Fuente & $\begin{array}{c}\text { Suma de } \\
\text { Cuadrados }\end{array}$ & GI & Cuadrado Medio & Razón-F & Valor-P \\
\hline A:Relación S/L & 95,7036 & 1 & 95,7036 & 10,88 & 0,0215 \\
B:Temperatura & 192,276 & 1 & 192,276 & 21,85 & 0,0055 \\
C:Tiempo & 6,35461 & 1 & 6,35461 & 0,72 & 0,4342 \\
AA & 2,14204 & 1 & 2,14204 & 0,24 & 0,6426 \\
AB & 0,081225 & 1 & 0,081225 & 0,01 & 0,9272 \\
AC & 2,0449 & 1 & 2,0449 & 0,23 & 0,6501 \\
BB & 18,02 & 1 & 18,02 & 2,05 & 0,2118 \\
BC & 0,119025 & 1 & 0,119025 & 0,01 & 0,9119 \\
CC & 37,0256 & 1 & 37,0256 & 4,21 & 0,0955 \\
Error total & 43,9961 & 5 & 8,79922 & & \\
Total (corr.) & 392,484 & 14 & & & \\
\hline
\end{tabular}


La tabla ANOVA particiona la variabilidad del porcentaje de reticulación de los taninos en piezas separadas para cada uno de los efectos. Probando la significancia estadística de cada efecto, comparando su cuadrado medio con un estimado del error experimental. Se comparó el valor-P de los respectivos experimentos con un nivel de significancia $\alpha=0,05$, donde se observó que los factores A (Relación S/L) y B (Temperatura) rechazan la hipótesis nula debido a que presenta un valor-P menor a 0,05 , lo que indica que son significativamente diferente de cero con un nivel de confianza del $95 \%$, es decir que los efectos A y $B$ si presentan influencia en el proceso de reticulación de los taninos. EL factor C (Tiempo) presenta un valor-P mayor a 0,05 por lo tanto se acepta la hipótesis nula y se concluye que no tiene efecto en el porcentaje de reticulación.
Para el caso de las interacciones entre los factores, tanto $A B, A C, y B C$ tienen un valor-P mayor a 0,05 por consiguiente se aceptan las tres hipótesis nulas, es decir, no hay efectos de las interacciones entre los factores A, B y C.

Se analizó el diagrama de Pareto (figura 1), donde se presentan en forma decreciente de importancia, cada uno de los efectos estudiados. En el diagrama de Pareto la longitud de cada barra es proporcional al efecto estandarizado, que es el efecto estimado entre su error estándar. La línea vertical que se presenta es utilizada para juzgar cuales son los efectos estadísticamente significativos. Cualquier barra que se extienda más allá de dicha línea vertical corresponde a efectos que son estadísticamente significativos con un $95 \%$ de nivel de confianza.

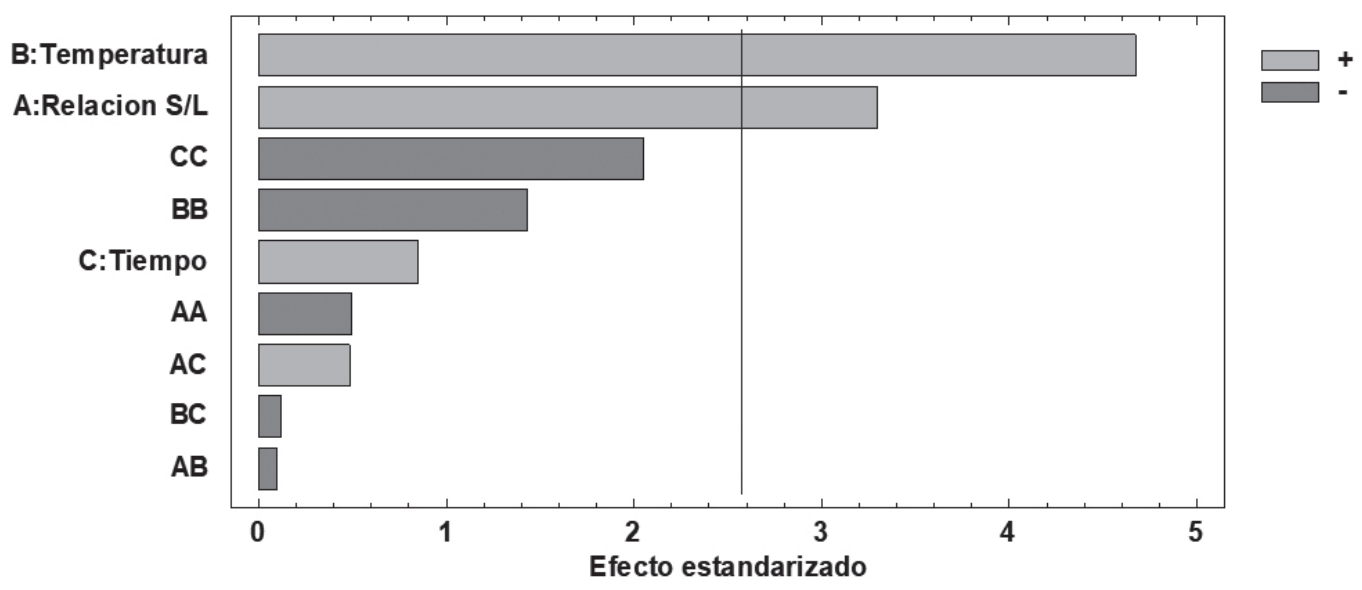

Figura 1. Diagrama de Pareto para la optimización de la reticulación.

En este caso los factores que sobrepasan la línea son $\mathrm{A}$ y $\mathrm{B}$, en consecuencia, son efectos significativos que influyen en la reticulación de los taninos, resultado que es comparable con el análisis de varianza. Los efectos pueden ser positivos o negativos según corresponda el signo. Para el caso de A y B indica que un aumento en la relación $\mathrm{S} / \mathrm{L}$ y la temperatura, aumentara el porcentaje de reticulación.

A continuación, se analizó los gráficos de efectos principales figura 2, análisis que permitió establecer los puntos óptimos para la reticulación de los taninos.

Como se observa en la figura 2, tanto la relación $S / L$ y la temperatura causan un crecimiento en el rendimiento de la reticulación. Por otro lado, el tiempo presenta un crecimiento hasta cierto punto, y seguidamente una disminución en el rendimiento. Este comportamiento se debe posiblemente que a mayor tiempo de reacción haya una descomposición de los taninos, y por ende una disminución en el porcentaje de rendimiento de la reticulación.

En el análisis de las interacciones de segundo orden, se tiene que no hay interacciones entre los factores analizados, estos resultados se pueden corroborar en la tabla 1, en donde el valor-P para las interacciones entre los factores $A B, A C$ y $B C$ es mayor a 0,05.

La función conllevó a un gráfico de superficie de respuesta, como se observa en la figura 3 , donde, con un tiempo de extracción de 10,4 horas, una relación $\mathrm{S} / \mathrm{L}$ de 0,499 y una temperatura de 95 ${ }^{\circ} \mathrm{C}$ se puede obtener un rendimiento óptimo de $73,81 \%$ de la reticulación de los taninos. 


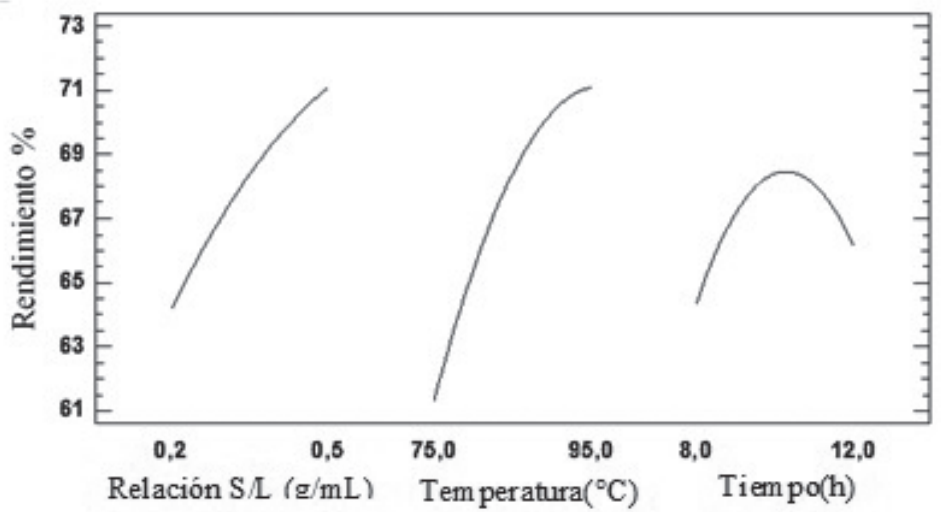

Figura 2. Gráfica de efectos principales para la reticulación de los taninos.

Al finalizar el análisis del diseño experimental y teniendo las condiciones de optimización de la reticulación, se realizaron ensayos por triplicado de esos puntos, se obtuvieron valores de rendimiento de $73,64 \% \pm 0,30$.

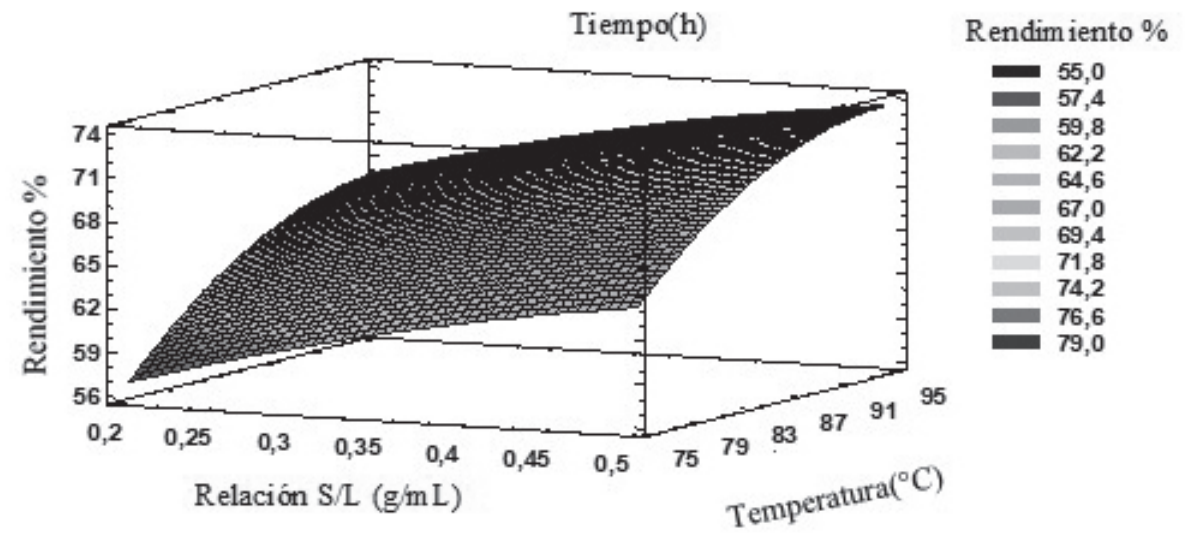

Figura 3. Gráfico de superficie de respuesta estimada.

Se utilizó un método de regresión no lineal para ajustar los datos experimentales a un modelo polinomial de segundo orden e identificar los términos relevantes del modelo, obteniéndose la ecuación 1.

$$
\begin{aligned}
\text { Rendimiento }= & -230,536+30,9963 \mathrm{~A}+4,36533 \mathrm{~B}+16,1779 \mathrm{C}-33,8519 \mathrm{~A}^{2}-0,095 \mathrm{AB}+ \\
& 2,38333 \mathrm{AC}-0,0220917 \mathrm{~B}^{2}-0,008625 \mathrm{BC}-0,791667 \mathrm{C}^{2}
\end{aligned}
$$

El proceso de reticulación de los taninos extraídos de la corteza, cuando se usa ácido sulfúrico concentrado como agente reticulante conduce a la formación de un material rígido de porosidad y tamaño de partícula adecuadas para los procesos de adsorción [11]. Es por ello que se analizaron los espectros IR de los taninos y una vez realizada la reticulación, cuyos resultados se presentan en la figura 4.
Los taninos presentaron una banda moderada entre 3400 y $3600 \mathrm{~cm}^{-1}$ correspondiente a las vibraciones de estiramiento del enlace $\mathrm{O}-\mathrm{H}$ de los compuestos fenólicos, característico en los taninos, al igual que bandas características de estiramiento $\mathrm{C}-\mathrm{H}$ a $2900 \mathrm{~cm}^{-1}$, el espectro IR de los taninos también mostró la banda de absorción a $1384,12 \mathrm{~cm}^{-1}$ que se asignan para la flexión $\mathrm{O}-\mathrm{H}$, el pico de $1638,52 \mathrm{~cm}^{-1}$ que se asigna a la 
vibración de estiramiento de los enlaces $\mathrm{C}=\mathrm{C}$ del anillo aromático. Después de la reticulación, se evidencia un incremento en la transmitancia en las bandas asociadas a los diferentes grupos $\mathrm{OH}$ fenólicos, el tratamiento acido conlleva a la apertura del heterociclo originando un carbocatión que puede ser capturado por otras unidades flavonoides presentes aumentando el número de grupos hidroxilo, es por eso que es evidente que hay una mayor transmitancia por ende aumenta el ancho de banda a $3452,19 \mathrm{~cm}^{-1}$, la aparición de una banda a $1471,88 \mathrm{~cm}^{-1}$ es un indicio de la exposición de $\mathrm{OH}$, debidos a que es una banda característica de la flexión de los grupos $\mathrm{OH}$ en el plano. Después de la reticulación, las bandas debido a $\mathrm{C}=\mathrm{O}$ se estiran y las vibraciones de estiramiento $\mathrm{C}=\mathrm{CO}$ se ampliaron y aparecieron como bandas moderadas, probablemente debido a la oxidación parcial de grupos hidroxilo [11]. Estos resultados indican que los grupos funcionales de los taninos sufrieron una modificación en la estructura debido a la reticulación, al ser tratadas con ácido, puede ocurrir hidrólisis o autocondensación. La degradación en medios ácidos conduce a la formación de catequinas y antocianidinas, este tipo de tratamientos con ácido conduce a la formación de estructuras que corresponden a taninos condensados[12].

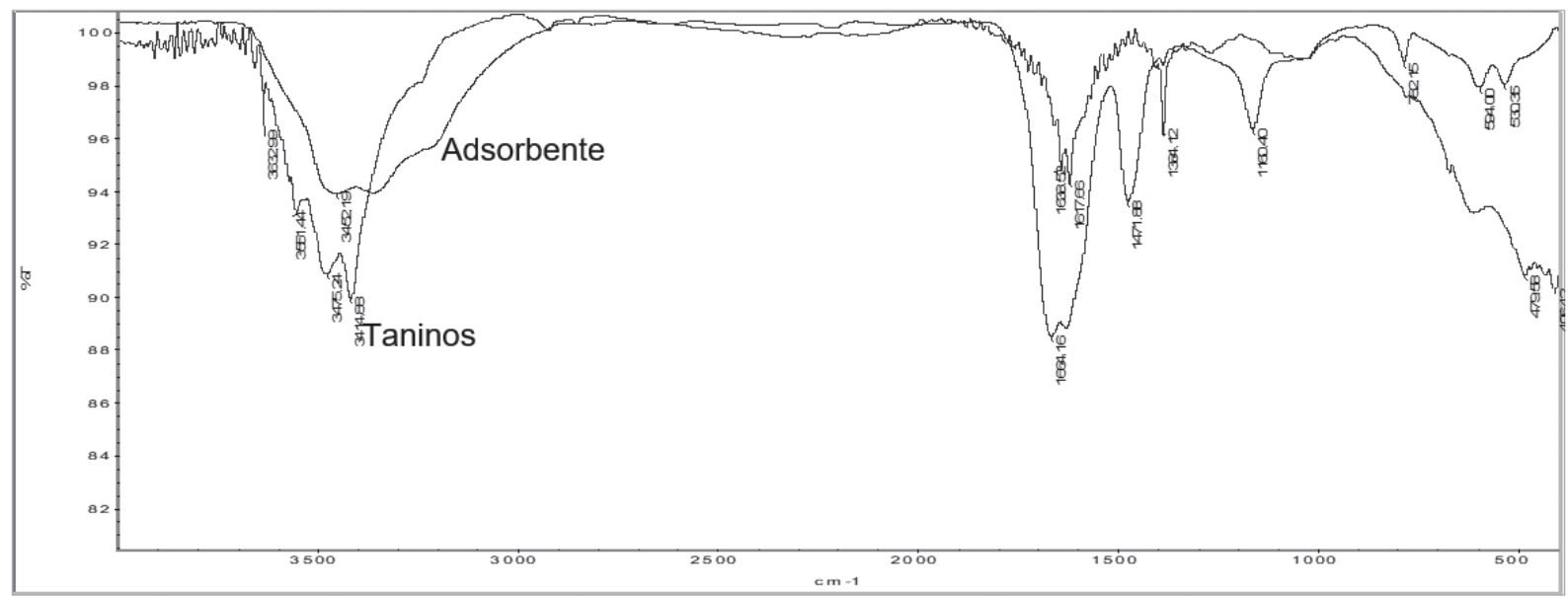

Figura 4. Espectros IR de los taninos y el adsorbente preparado.

Se realizaron experimentos para determinar el área superficial mediante el método BET. Los resultados obtenidos por BET mostraron un área de $4,17 \mathrm{~m}^{2} \mathrm{~g}^{-1}$, dicho valor es comparable con adsorbentes a base de biomasa que presentan valores de 5,91 $\mathrm{m}^{2} \mathrm{~g}^{-1}$ [17], por otro lado un trabajo realizado por Alegria (2017) donde se realiza una modificación química a los taninos naturales extraídos de la corteza de Pinus patula arrojó valores de 9,8 $\mathrm{m}^{2} \mathrm{~g}^{-1}$ [15].

\section{Conclusiones}

El porcentaje de humedad y cenizas de la corteza de Pinus Patula arrojaron valores de $26,27 \%$ y $1,77 \%$ respectivamente, estando dentro de los valores reportados para la biomasa vegetal. Se logró extraer los taninos de la corteza de Pinus patula con un porcentaje de extracción del $59,04 \%$, y su caracterización se realizó por espectroscopia IR, identificando los grupos característicos presentes en los extractos tánicos, dichas bandas a 3400 y $3600 \mathrm{~cm}^{-1}$ correspondiente a las vibraciones de estiramiento del enlace $\mathrm{O}-\mathrm{H}$ de los compuestos fenólicos, así mismo la banda a 1638,52 $\mathrm{cm}^{-1}$ que se asigna a la vibración de estiramiento de los enlaces $\mathrm{C}=\mathrm{C}$ del anillo aromático.

A través del diseño de superficie Box-Behnken, se logró establecer que el máximo rendimiento de la reacción de reticulación fue de $73,81 \%$ con unas condiciones de optimización de relación $\mathrm{S} / \mathrm{L}$ de $0,499 \mathrm{gmL}^{-1}$, temperatura de $95{ }^{\circ} \mathrm{C}$ y un tiempo de reacción de 10,45 horas. De esta manera se establecieron las condiciones óptimas para la reacción de reticulación y así mejorar las características del adsorbente, insolubilidad, aumento en el número de grupos hidroxilo. Por medio de la caracterización de espectroscopía IR se tuvo certeza de la modificación de los taninos a través de la reticulación con ácido sulfúrico 
concentrado, conllevando a la formación de catequinas y antocianidinas.

\section{Agradecimientos}

Agradecera los docentes del Grupo de Investigación en Química Analítica Ambiental (GIQA), al grupo de Química de productos naturales (QPN) y al departamento de Química de la Universidad del Cauca por apoyar esta investigación.

\section{Referencias bibliográficas}

[1] Serret Guasch N, Giralt Ortega G, Quintero Ríos M. Caracterización de aserrín de diferentes maderas. Tecnol. Química. 2016;36(3):395-504.

[2] Alvarez Godoy E. Aprovechando los Residuos Madereros (sitio en internet). Ecoportal. Disponible en: https://www.ecoportal.net/ temas-especiales/educacion ambiental/ aprovechando_los_residuos_madereros/. Acceso el 18 de julio 2020.

[3] Merle C, López O. Estudios de Economía forestal en el marco de la Misión de Crecimiento Verde en Colombia (sitio en internet). Disponible en: https://www.dnp. gov.co/Crecimiento-Verde/Documents/ejestematicos/forestal/Productos finales/Resumen V2018-09-30.pdf. Acceso 14 de julio 2020.

[4] Gómez EA, Ríos LA, Peña JD. Madera, un Potencial Material Lignocelulósico para la Producción de Biocombustibles en Colombia. Inf. Tecnológica. 2012;23(6):73-86.

[5] Choudhary BC, Paul D, Borse AU, Garole DJ. Surface functionalized biomass for adsorption and recovery of gold from electronic scrap and refinery wastewater. Purif. Technol. 2018;195:260-270.

[6] Bacelo HA, Santos SC, Botelho CM. Tanninbased biosorbents for environmental applications - A review. Chem. Eng. J. 2016;303:575-587.

[7] Ogata T, Research YN. Mechanisms of gold recovery from aqueous solutions using a novel tannin gel adsorbent synthesized from natural condensed tannin. Water Res. 2005;39(18):4281-4286.

[8] Al-Qodah Z, Yahya A, Al-Shannag M. On the performance of bioadsorption processes for heavy metal ions removal by lowcost agricultural and natural by-products bioadsorbent: a review. Desalin Water Treat.
2017;85:339-357.

[9] Kavitha UV, Kandasubramanian B. Tannins for wastewater treatment. SN Appl. Sci. 2020;2(6):1081.

[10] Santos SC, Bacelo HA, Boaventura RA, Botelho CM. Tannin-Adsorbents for Water Decontamination and for the Recovery of Critical Metals: Current State and Future Perspectives. Biotechnol. J. 2019;14(12):1900060.

[11] Gurung M, Adhikari BB, Kawakita H, Ohto K, Inoue K, Alam S. Recovery of Au(III) by using low cost adsorbent prepared from persimmon tannin extract. Chem. Eng. J. 2011;174(2):556563.

[12] Arbenz A, Avérous L. Chemical modification of tannins to elaborate aromatic biobased macromolecular architectures. Green Chem. 2015;17(5):2626-2646.

[13] Sarria Villa RA, Corredor JA, Isabel Paez M. Isolation of Catechin and Gallic Acid from Colombian Bark of Pinus patula. Chem. Sci. J. 2017;8(4):174.

[14] Ospina Penagos CM, Hernández Restrepo RM, Rincón EA, Sánchez Ocampo FA, Urrego Mesa JB, Rodas Peláez C, et al. Guías silviculturales para el manejo de especies forestales con miras a la producción de madera en la zona andina colombiana. (Sitio en internet). CENICAFE. Disponible en: http:// cia.corantioquia.gov.co/cgi-bin/koha/opacdetail.pl?biblionumber=11823: Acceso el 26 de agosto 2020.

[15]Alegria K. Determinación de la capacidad de adsorción de la resina de Pinus Patula modificada químicamente para retener cromo de efluentes generados por la actividad de curtiembres. (Tesis pregrado). Popayán, Colombia: Universidad del cauca; 2017.

[16] O. Saravia JM, Cano T, Chavez, B, Cerezo E. Extracción y caracterización de taninos en corteza de 3 especies forestales cultivadas en Guatemala, pino ocote (Pinus oocarpa schiede), Encino negro (Quercus brachystachys benth) y aliso común (Alnus jorulensis hbk.). Una alternativa de desarrollo agroindustrial. (Tesis maestria). Guatemala. Instituto de investigaciones agronómicas. 2002.

[17]Rosángeles Hernández ME, Carrasco $P$, Mujica R. Evaluación de la capacidad de adsorción de desechos agroindustriales para la remoción de ácido acético. Rev. Fac. Ing., Univ. Cent. Venez. 2007;22(3):31-46. 